\title{
On the behavior of stellar rotation in the solar neighbourhood
}

\author{
Daniel B. de Freitas and J. R. De Medeiros \\ Departamento de Física, Universidade Federal do Rio Grande do Norte, \\ 59072-970 Natal, RN, Brazil \\ email: danielbrito@dfte.ufrn.br
}

\begin{abstract}
Rotation is a fundamental physical parameter in stellar astrophysics, playing an important role on the formation and evolution of stars. This parameter may also offer valuable information on stellar magnetism, mixing in the stellar interior, tidal interactions in close binary, as well as on angular momentum transfer and rotational breaking due to planets. The present work brings the results of an unprecedented study on the behavior of the distribution of the projected rotational velocity $(v \sin i)$ as a function of galactic position, on the basis of an unique sample of 14000 main-sequence field stars, along the spectral regions $\mathrm{F}$ and $\mathrm{G}$. The vsini measurements used in this analyses were obtained from observations carried out with the CORAVEL spectrometers, with a precision better than about $1 \mathrm{~km} / \mathrm{s}$.
\end{abstract}

Keywords. stellar rotation, solar neighbourhood, galactic coordenates.

\section{Introduction}

One of the most puzzling questions in stellar astrophysics is the possible link of rotation with the location of stars in the Galaxy. This study is relevant to a variety of problems in stellar astrophysics, particularly for the understanding of the star formation process itself. Different studies have found no dependence between projected rotational velocity vsini and galactic coordinates or have pointed for such a dependence for specific group of stars (Burki \& Maeder 1977; Guthrie 1982; Wolff et al. 1982, De Medeiros et al. 2000). In the present study we revisit the question of the link between rotation and galactic coordinates, now on the basis of the largest sample of stars used for such an analyses, complete in visual magnitude and kinematically.

\section{Working sample}

Our study is based on the complete, magnitude-limited, and kinematically unbiased sample of nearby $\mathrm{F}$ and $\mathrm{G}$ dwarf stars from the Geneva-Copenhagen survey of the solar neighbourhood, carried out by Nordstrm et al. (2004) and Holmberg et al. (2007). These authors bring age, metallicity, projected rotational velocity $\langle v \sin i\rangle$ and kinematic properties for about $14000 \mathrm{~F}$ and $\mathrm{G}$ dwarfs. For the large majority of the stars (12 941 stars) the $\langle v \sin i\rangle$ data were obtained from observations with the photoeletric cross-correlation spectrometers CORAVEL (see Baranne et al. 1979; Mayor 1985), by applying the calibrations of Benz \& Mayor $(1980,1984)$. Such a procedure gives $\langle v \sin i\rangle$ values with a precision of $1 \mathrm{~km} / \mathrm{s}$, at least for rotations lower than about $30 \mathrm{~km} / \mathrm{s}$ De Medeiros \& Mayor 1999. As shown by Nordstrm et al. (2004) the large majority of their programme stars have rotations below $20 \mathrm{~km} / \mathrm{s}$. 

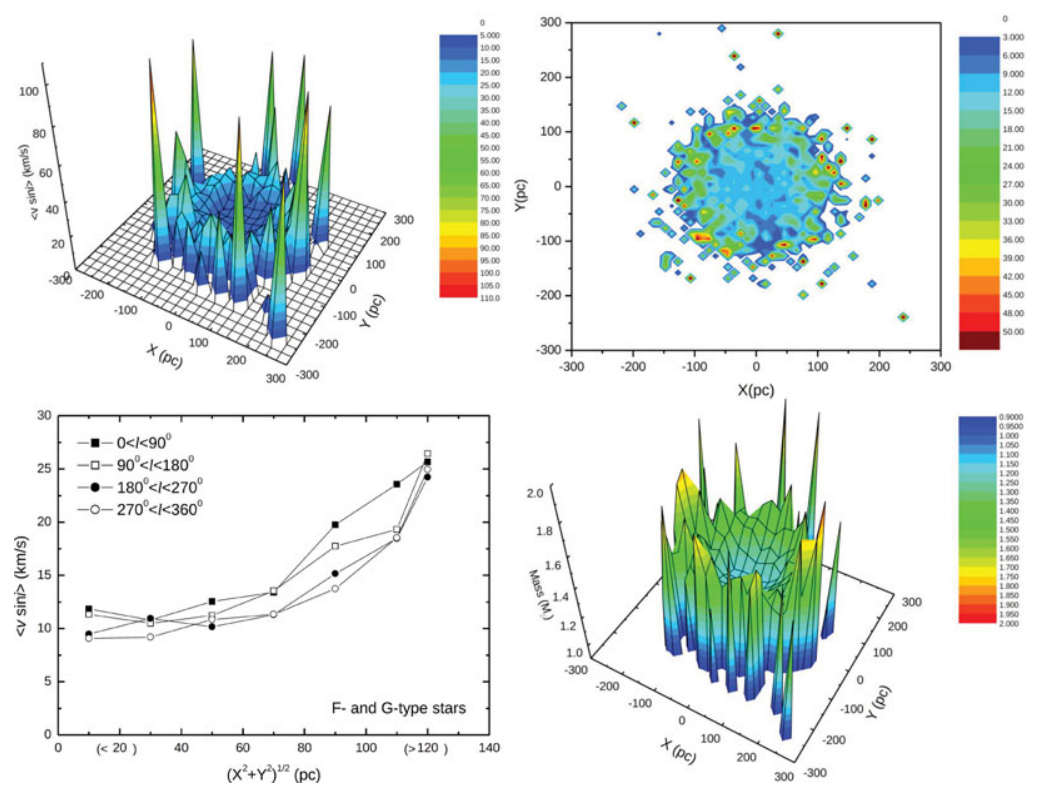

Figure 1. Upper panels: Distribution of the average rotational velocity $\langle v \sin i\rangle$ in the galactic plane for F- and G-type stars (left) and contour diagram showing several level curves in the $\mathrm{XY}$ galactic plane corresponding to several values of $\langle v \sin i\rangle$ in a resolution of $5 \mathrm{X} 5 \mathrm{pc}^{2}$ (right). Lower panels: Longitudinal distribution of $\langle v \sin i\rangle$ as a funtion of the XY galactic plane for all stars of the sample (left) and distribution of the average mass $\left\langle M / M_{\odot}\right\rangle$ in the galactic plane (right).

\section{Results and Conclusions}

In Fig. 1, we show the main results from this study. From this figure, we can conclude that: i) there is a clear dependence of rotation on the stellar galactic position for single stars in the solar neighbourhood, in the sense that, high values of $\langle v \sin i\rangle$ concentrate on locations further away of the Sun and low values close to it (see upper panel plots); ii) there is a positive gradient of $\langle v \sin i\rangle$ relative at the distance from star to $\operatorname{Sun}\left(X^{2}+\right.$ $\left.Y^{2}\right)^{-1 / 2}$; iii) there is also a dependence of rotation with the galactic longitude $l$, in the sense that high values of $\langle v \sin i\rangle$ are located towards the galactic anticenter (see lower left panel plot) and; iv) there is a similar behavior for stellar mass (see lower right panel plot) and metallicity (not shown).

\section{References}

Baranne, A., Mayor, M., \& Poncet, J. L., 1979, Vistas in Astron. 23, 279

Benz, W. \& Mayor, M., 1980, A\&A, 93, 235

Benz, W. \& Mayor, M., 1984, A\&A, 138, 183

Burki, G. \& Maeder, A., 1977, A\&A, 57, 401

De Medeiros, J. R. \& Mayor, M. 1999, A\&AS, 139, 433

De Medeiros, J. R., Carvalho, J. C., Soares, B. B., Da Rocha, C., \& Maia, M. R. G., 2000, A\&A, 358,113

Guthrie, B. N. G., 1982, MNRAS, 198, 795

Holmberg J., Nordstrom B., \& Andersen J., 2007, A\&A, 475, 519

Mayor, M. 1985, in Stellar Radial Velocities, ed. A.G.D. Philip \& D. W. Latham (Schenectady,

New York: L. Davis Press) IAU Coll., 88, 21

Nordstrom, B., Mayor, M., \& Anderson, J., et al. 2004, A\&AS, 418, 989

Wolff, S. C., Edwards, S., \& Preston, G. W., 1982, ApJ, 252, 322 